\title{
Characterization of drug authenticity using thin-layer chromatography imaging with a mobile phone
}

Hojeong $\mathrm{Yu}^{\mathrm{a}, \mathrm{b}, \#}$, Huy M. Le ${ }^{\mathrm{a}, \mathrm{c}, \#}$, Eliangiringa Kaale ${ }^{\mathrm{d}}$, Kenneth. D. Long ${ }^{\mathrm{b}, \mathrm{e}}$, Thomas Layloff ${ }^{\mathrm{f}}$, Steven S. Lumetta ${ }^{\mathrm{a}, \mathrm{c}}$, Brian T. Cunningham ${ }^{\mathrm{a}, \mathrm{b}, \mathrm{e}, *}$

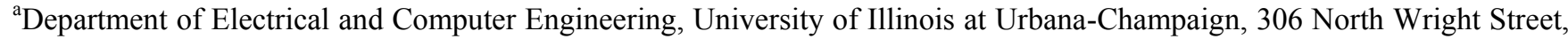
Urbana, Illinois 61801, USA

${ }^{\mathrm{b}}$ Micro and Nanotechnology Laboratory, University of Illinois at Urbana-Champaign, 208 North Wright Street, Urbana, Illinois 61801, USA

${ }^{\mathrm{c}}$ Coordinated Science Laboratory, University of Illinois at Urbana-Champaign, 1308 West Main Street, Urbana, Illinois 61801, USA

${ }^{\mathrm{d}}$ Pharm. R\&D Lab, School of Pharmacy, Muhimbili University of Health and Allied Sciences, 11103 Upanga West, Dar Es Salaam, Tanzania

${ }^{\mathrm{e}}$ Department of Bioengineering, University of Illinois at Urbana-Champaign, 1304 West Springfield Avenue, Urbana, Illinois 61801, USA

${ }^{\mathrm{f}}$ Management Sciences for Health, 4301 N. Fairfax Drive, Suite 400, Arlington, Virginia 22203, USA

${ }^{*}$ Corresponding author

Tel.: +1 2173332301

E-mail: bcunning@illinois.edu

${ }^{\#}$ These authors contributed equally.

\section{Graphical abstract:}
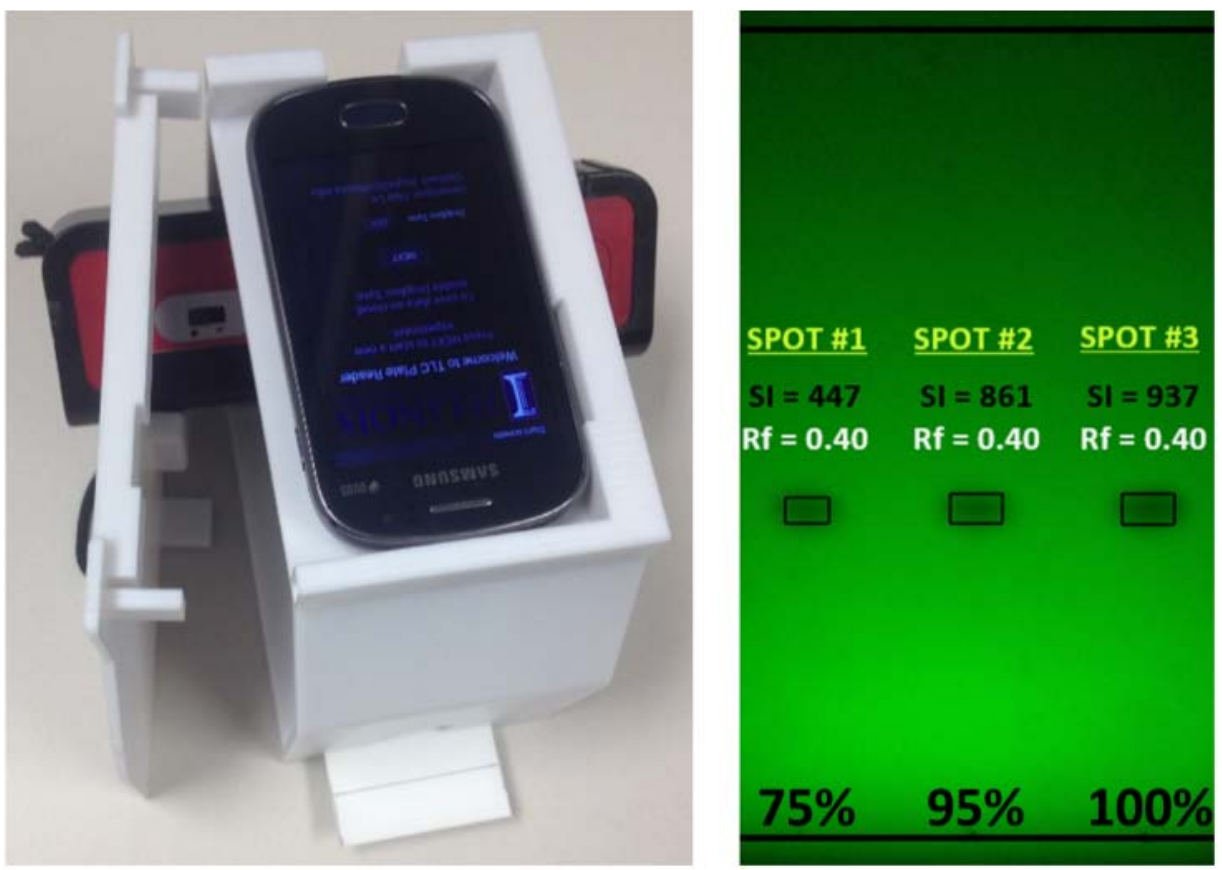

(C) 2016. This manuscript version is made available under the Elsevier user license 


\section{Abstract}

Thin-layer chromatography (TLC) has a myriad of separation applications in chemistry, biology, and pharmacology due to its simplicity and low cost. While benchtop laboratory sample application and detection systems for TLC provide accurate quantitation of TLC spot positions and densities, there are many applications where inexpensive and portable instruments would greatly expand the applicability of the technology. In this work, we demonstrate identity verification and concentration determination of pharmaceutical compounds via TLC using a custom 3D-printed cradle that interfaces with an ordinary mobile phone. The cradle holds the mobile phone's internal, rear-facing camera in a fixed position relative to a UV lamp and a TLC plate that includes a phosphor in the stationary phase. Analysis of photographs thus reveals the locations and intensities of principal spots of UV-absorbing drugs. Automated image analysis software determines the center location and density of dark spots, which, using integrated calibration spots of known drug compounds and concentrations, can be used to determine if a drug has been diluted or substituted. Two independent image processing approaches have been developed that may be selected based upon the processing capabilities of the smartphone. Each approach is able to discern 5\% drug concentration differences. Using single-component solutions of nevirapine, amodiaquine, and paracetamol that have been manually applied, the mobile phone-based detection instrument provides measurements that are equivalent to those obtained with a commercially available lab-based desktop TLC densitometer.

Keywords: Thin-layer chromatography; Pharmaceutical compound analysis; Mobile phone sensing; Densitometry; Falsified drug detection

\section{Introduction}

Chromatography-based methods for separating, detecting and quantifying active pharmaceutical ingredients (APIs) in chemical mixtures through their components' unique partition coefficients are fundamental to the field of analytical chemistry [1-3]. One specific application for chromatography with important implications for preserving human health is identification of falsified and substandard pharmaceutical products [4-6]. The sale of falsified and substandard drugs is currently a multi-billion dollar industry, which thrives especially in developing countries. According to the World Health Organization, falsified and substandard drugs account for up to $30 \%$ of sales of pharmaceuticals in developing markets [7, 8]. Such products often are produced by highly-organized unscrupulous manufacturers who undertake efforts to replicate the physical characteristics such as shape, color, and packaging of authentic drug products, but with the active ingredient either missing, diluted or completely replaced with another API. These substandard or falsified products pose severe health risks for patients who receive doses of an API that are lower than that required for efficacy, or receive a substitute API that has no therapeutic indication for the disease of concern. The most common incidences of falsified critical drugs are encountered in the treatment of malaria, HIV/AIDS, tuberculosis, and cancer, thus these products pose an extreme health risk to the patients who take them. An estimated 100,000 deaths per year have been linked to the falsified and substandard drug trade in Africa [7-9]. Thus, there is an urgent need for an efficient and low-cost technology for identification of these products in the drug supply chain. Ideally, such a detection system can be combined with an information-sharing network that will enable falsified and/or substandard products to be rapidly identified and flagged by their source, batch number, packaging, and physical appearance. 
A variety of chromatographic techniques are used for analysis of falsified and substandard medicines and illegal pharmaceutical preparations today. Thin-layer chromatography (TLC), liquid chromatography (LC), gas chromatography (GC), and chromatographic fingerprinting are used widely to separate and quantify the chemical compounds present in dosage forms, and to subsequently differentiate falsified and substandard products from authentic products. Among these approaches, TLC represents the simplest and lowest cost approach that is most amenable to adoption in developing parts of the world, where expensive laboratory instruments, highly trained staff members, and analytical laboratories are not readily available. The physical principles of TLC separation have been thoroughly studied [10, 11], and are summarized in Figure 1S. Testing of an unknown drug compound may be performed by comparison of a TLC profile of the unknown compound with calibration profiles. To reduce sources of error, we include two such profiles on the same plates as the sample under test. For example, aliquots of a drug compound prepared at full concentration (100\%) and at a known dilution, e.g., 75\% of full concentration [12-14], are placed next to an aliquot of an unknown concentration. If a drug has been diluted with respect to the full concentration drug, the spot intensity is reduced because the amount of ultraviolet (UV) light absorbed is directly proportional to the amount of drug present. Thus, direct comparison of the spot intensity for the unknown concentration with the spot intensity of the calibration samples can be used to estimate the concentration of an unknown drug. Similarly, the vertical position of a spot in a TLC image can be used to verify the identity of a drug in an unknown sample because different chemical compounds ascend the plate at different rates.

With the advent of inexpensive digital cameras, image-based densitometric evaluation of TLC plates has become prevalent [15-17], using desktop instruments that interface with a personal computer. Commercial instruments incorporating flatbed scanners, CMOS cameras, white light illumination, and high intensity UV lamps have been developed and widely adopted, with prices in the range of US \$5,000 - 45,000 [18-20]. A large number of applications for an inexpensive handheld TLC scanning and image analysis system exist for detection scenarios in food safety, pharmaceutical safety, manufacturing quality control, and water quality testing in which an immediate result is desired at the point of testing - particularly when a centralized laboratory facility is not available.

Since the introduction of smartphones in 2007, the capabilities of mobile technology have rapidly increased in terms of computational power, prevalence of internet connectivity, and integration of image sensors with high pixel counts. Advances in mobile technology have been accompanied by decreased manufacturing costs, enabling the rapid adoption of smartphones to the point that nearly $1.6 \mathrm{~B}$ people are estimated to use a smartphone [21], with 2014 sales exceeding $1.2 \mathrm{~B}$ units [22]. For these reasons, smartphones are rapidly being adapted for applications that include healthcare, microscopy, and food safety, deriving from the ability to perform assays for disease biomarker detection [23, 24], label-free biosensor measurement [25], fluorometric sensing/imaging [26, 27], interpretation of paper test strips [28], and many others [29, 30]. The multi-core processors of the most modern smartphones are sufficiently fast to perform on-board image and data processing, while cloud-based services can be readily established to log the measurements from a community of users, to seek trends in the gathered data, and to communicate the results of analysis back to users in real-time. Practical tradeoffs in connectivity, speed, and power consumption must be considered when deciding upon the extent to which processing is performed directly on the smartphone versus sending data to a remote computer.

The system employed in this work is comprised of a 3D-printed plastic cradle, a Samsung Galaxy Fame smartphone, and a battery-powered UV lamp. The cradle holds the phone fixed relative to the 6V UV lamp, enabling capture of $2560 \times 1920$ pixels (5M pixels) images of TLC plates illuminated by $254 \mathrm{~nm}$ light. A conventional $100 \times 50 \mathrm{~mm}$ TLC plate is inserted into the cradle through a narrow slot. The system is compact (Figure $2 \mathrm{~S}$ ), lightweight (700 g, including the smartphone and 
the UV lamp with four 1.5V AA batteries), and inexpensive (3D-printed cradle: US \$130, handheld UV lamp: US \$63, smartphone: US \$59.99).

While an inexpensive mobile device was specifically selected for this application based on its affordability for applications in drug authenticity screening in resource-limited areas, the approach demonstrated in this work may be easily adapted to any mobile device with an internal, rear-facing camera. In addition to the detection instrument hardware, we have developed a software application that includes an image processing algorithm that extracts both vertical location and intensity of three different spots on a TLC plate. For this initial demonstration, we focused our efforts upon TLC analysis of single-component drugs, and the ability of our approach to differentiate full-concentration drugs from those that have been diluted to $95 \%$ and $75 \%$ of their full concentration. $75 \%$ purity is the lowest limit of drug concentration considered acceptable for a single tablet, while many fraudulent drug formulations are known to have significantly lower concentrations. In this work, we evaluate and compare two approaches for data analysis.

First, we developed a software application containing an internal image processing algorithm that runs efficiently on the smartphone by partitioning every pixel of the TLC plate image into one of two classifications (spot or non-spot) and analyzing the absorption coefficient of the fluorescence only within the spot locations. Alternatively, a personal computer executable program based on an external image processing algorithm that operates on a separate computer running Matlab software analyzes the images transferred from the smartphone. The external algorithm computes spot intensity by integrating pixel values $(P V \mathrm{~s})$ in an area of analysis. . The retention factor $\left(R_{f}\right)$ is defined as the distance between the center of a spot and the origin line normalized to the distance between the solvent front line and the origin line. Both image processing approaches require a reference image of a blank TLC plate to normalize the effects of non-uniform illumination from the UV lamp. The algorithm running in an external computer needs one blank TLC image that is pre-stored in the computer for every measurement, while the algorithm running in the smartphone requires an image of a blank TLC plate to be taken before each assay. Both methods were compared directly to measurements taken with a commercially available densitometer (TLC Scanner 3; CAMAG, Muttenz, Switzerland) for a set of TLC plates prepared with 100\%, 95\%, and 75\% concentrations of nevirapine (an anti-retroviral product), amodiaquine (an anti-malarial and anti-inflammatory product), and paracetamol (an anti-inflammatory product). Our results show that, using either image processing method, the smartphone-based TLC system provides measurements for retention factor and spot intensity that are equivalent to those taken with the commercial instrument.

\section{Materials and methods}

The drugs selected for this study are representative of those occasionally targeted by fraudulent drug manufacturers and that represent treatments for which severe health consequences result for patients that inadvertently take a dose lower than prescribed. Paracetamol is a pain reliever and a fever/inflammatory reducer used to treat conditions including osteoarthritis, spinal pain, and headache [31]. Nevirapine is a first-generation non-nucleoside reverse-transcriptase inhibitor used for the treatment and management of HIV/AIDS [32]. Amodiaquine is a drug used for malaria treatment [33]. All three drugs are included in the WHO list of essential medicines [34]. Each drug is sold in tablet form, and contains a single API that absorbs UV light [35]. Thus, during TLC analysis, each drug is expected to produce only a single spot during plate development, and the presence of additional spots indicates the presence of undesirable components. Chemicals and reagents are briefly described in the supplementary information. 


\subsection{Preparation of Sample Stock and Working Solutions}

The procedures used for performing the TLC spotting and development are described in the Global Pharma Health Fund Minilab ${ }^{\circledR}$ manuals [36]. One tablet (paracetamol $500 \mathrm{mg}$, amodiaquine $200 \mathrm{mg}$, or nevirapine $200 \mathrm{mg}$ ) was ground into a fine powder while wrapped in aluminum foil, and the powder was transferred into a $100 \mathrm{~mL}$ volumetric flask containing $40 \mathrm{~mL}$ of methanol. The flask was shaken for three minutes to dissolve the API and was set to stand for approximately five minutes to allow the undissolved residue to settle leaving a clear supernatant liquid. Appropriate amounts of methanol were then added to the flask to obtain the target of $5 \mathrm{mg} / \mathrm{ml}$. Aliquots were drawn from the supernatant stock sample solutions to prepare $100 \%, 95 \%$, and $75 \%$ solutions (Table $1 \mathrm{~S}$ ).

\subsection{Chromatography and Densitometric Scanning}

The solvent front line was pre-marked at $70 \mathrm{~mm}$ from the bottom of the TLC plate. Two microliters of the test solutions were manually placed on the plate along the origin line as a spot using precision 2-microliter capillaries. After spotting, the plates were air-dried and then placed into the developing jar. The developing jar was lined with filter paper on one long side to aid saturation, and the mobile phase was poured into the jar. The composition of the mobile phase for each drug is shown in Table 2S. The jar lid was closed for 20 minutes to saturate the tank before the plate was developed. After the plate was placed into the jar and the mobile phase front proceeded to the pre-marked solvent front line, the plate was removed and allowed to dry for three to five minutes. After drying, the plates were scanned using the conventional densitometer (UV illumination: $\lambda_{\text {peak }}=254 \mathrm{~nm}$ ). The detector slit parameters were set at $6 \times 0.45 \mathrm{~mm}$, the scanning speed was set at $20 \mathrm{~mm} / \mathrm{s}$, and the data resolution was set at $100 \mu \mathrm{m} / \mathrm{step}$. The data capture and calculations obtained from the scanned plates were performed with the instrument-supplied planar chromatography manager software (winCATS 1.4.3; CAMAG).

\subsection{Smartphone TLC Analyzer}

A schematic of the system and a photo of the captured image are shown in Figure 1. A custom-designed cradle that is fabricated using a 3D-printer (Objet350 with polyjet material Vero; Stratasys, MN, USA) aligns the smartphone (S6812; Samsung, Seoul, South Korea), the UV lamp (254 nm Handheld Mini UV Lamp; UVP, CA, USA), and the TLC plate. The smartphone's rear-facing camera is aligned with a plate guide that places the TLC plate into focus and into the camera field of view (Figure 3S). The TLC plates are inserted into the cradle body via a small entrance slit placed in front of the cradle, and a guide holds the plate down flat. The distance from the camera to the plate is $100 \mathrm{~mm}$. The cradle provides a dark environment that prevents external light sources from degrading the integrity of the TLC images. The UV lamp is removable and is installed using the positioning holes of the cradle body and lid to fix it securely in place. The UV lamp is oriented at a $39^{\circ}$ angle with respect to the TLC plate surface in order to provide UV illumination over a wide surface area, although, due to the intrinsic geometry of the lamp, the illumination intensity is greatest along the central axis, and falls off smoothly as one moves away from the central axis. The spots formed by drugs on the TLC plates cannot be observed without UV light (Figure 2(a)). However, as Figure 2(b) shows, when the plate is illuminated with the UV light, the fluorescent compound emits green light that is reduced in intensity by the UV-absorbing APIs, resulting in dark spots on a bright green background. 
Figure 2(c) depicts the fluorescent intensity distribution over the plate. The area of image analysis is approximately $40 \times 80$ $\mathrm{mm}$, which is comprised of $1020 \times 2060$ pixels, enabling the entire plate area beyond the origin line to be analyzed.

\subsection{Image Processing: External Algorithm}

An unprocessed RGB (red, green, blue) photo taken by the smartphone is comprised of a matrix of $2560 \times 1920 \times 3$ pixels (the smallest resolvable image elements). The first two dimensions represent the size of the photo, and the last dimension provides a 24-bit RGB color space. Each of the RGB intensity components of each pixel is stored as an 8-bit unsigned integer, giving a range of 0 to 255. A flow chart of the automated processing is shown in Figure 4S. First, data acquisition is performed when a user initiates a scan, for which a sequence of 10 nominally identical images of the plate is acquired in series (1 photo / $1 \mathrm{sec}$, ISO 400, exposure -4). Compared to colorimetric analysis using each RGB channel independently, we analyze the total light intensity, as the fluorescent emission of the TLC plate is comprised of only a narrow band of wavelengths in the green channel. To extract the green fluorescence intensity levels from a color image, we apply a colormap transformation to change the color image in its RGB color space to its representation in YIQ color space that is more sensitive to changes in luminosity by the following transform [37].

$$
\left[\begin{array}{l}
Y=0.2989 R+0.5866 G+0.1144 B \\
I=0.5959 R-0.2741 G-0.3218 B \\
Q=0.2113 R-0.5227 G+0.3113 B
\end{array}\right]
$$

In the YIQ system, the $Y$-channel carries information of perceived relative brightness of different colors, while $I$ - and $Q$-channels are jointly related to the hue and saturation components of a color space. We select the $Y$-channel image represented by a $2560 \times 1920 \times 1$ matrix for the TLC plate analysis because the light intensity variation due to the existence of spots on the TLC plate is mostly captured in the $Y$-channel. Next, $10 Y$-channel images are averaged to generate a single image (Figure 4S(a-c)). Images captured via CMOS sensors suffer from various noise sources, including shot noise, thermal noise, and readout noise, that can generate undesired standard deviation for low-intensity images if only a single image is used [38]. Image averaging reduces the impact of these noise sources without compromising spatial detail of the original image because it increases an image's signal to noise ratio (SNR) [39]. As shown in Figure 4S(d), the stored Y-channel image of a blank TLC plate is subtracted from the averaged $Y$-channel image to remove the effect of uneven illumination resulting from the irregular spatial intensity distribution of UV light and the tilted orientation of the lamp. The TLC plate markings (the solvent front line and the origin line) are also easily distinguished after the subtraction and can be located by our image processing algorithm, which measures the minimum pixel value when scanned in the vertical direction with a 10pixel offset from both sides, while avoiding the spots (Figure 4S(e)). The analysis area for three different spots is defined as a rectangular box whose edges are matched to a threshold value $\left(T_{h}\right), 0.45$, around a spot of $100 \%$ drug concentration (Figure $4 \mathrm{~S}(\mathrm{f}-\mathrm{g})$ ). The threshold value is set by considering overall background noise, where the largest nonspecific noise observed has a pixel intensity ranging from 0.5 (green pixel) to 1 (red pixel). We set 0.05 (10\% of 0.5 ) of extra margin to improve the robustness of classifying a pixel as either "spot contributed $(P V \leq 0.45)$ " or "background noise $(P V>0.45)$ ". The analysis area is well matched with the relevant size of the full concentration spot, and a center of the analysis area is considered as the center of the spot. The retention factor $R_{f}$ of each spot is then measured as the ratio of the vertical distance traveled to the distance between the solvent front and origin lines, as shown in the following equation: 


$$
\begin{gathered}
\quad R_{f}=\frac{\text { Distance traveled by the principal spot }}{\text { Distance traveled by the solvent front }} \\
=\frac{\# \text { of pixels between the spot's center and the origin line }}{\# \text { of pixels between the solvent front line and the origin line }}
\end{gathered}
$$

The process for characterizing the spot intensity is shown in Figure 4S(h). Among the pixels within the analysis domain, those with values below the $T_{h}$ are selected as constituting a "principal spot" first. Then, to remove nonspecific singular noise, pixels with values less than a 10\%-offset cutoff are ignored because several pixels reach to very small $P V$ occasionally and they contribute to an inaccurate spot intensity computation. This cutoff value is defined by:

$$
10 \% \text {-offset }=\min . P V+\left(T_{h}-\min . P V\right) / 10
$$

where the min. $P V$ is the absolute minimum pixel value within the analysis domain. The pixel values that lie within the band, between the $T_{h}$ and the cutoff, are integrated in order to have the spot intensity described by the equation:

$$
\text { Spot intensity } \left.=\sum_{j=p_{y 1}}^{p_{y 2}} \sum_{i=p_{x 1}}^{p_{x 2}} P V_{i, j} \quad \text { if cutoff } \leq P V_{i, j} \leq T_{h}\right)
$$

where the $p_{x l} / p_{x 2}$ are pixel indices corresponding to the both vertical edges of the analysis area, while the $p_{y l} / p_{y 2}$ are pixel indices corresponding to the both horizontal edges. All three spots are analyzed using the approach described (Figure $4 \mathrm{~S}(\mathrm{i})$ ).

\subsection{Image Processing: Internal Algorithm}

The flow chart of the internal algorithm is shown in Figure 5S. For detecting lines and segmenting out spots, luminance matters most, as described in the External Algorithm section. The internal algorithm thus follows the same first steps (Figure $4 \mathrm{~S}(\mathrm{a}-\mathrm{c})$ ) to compute an average luminance $(Y)$ image (1 photo / $1 \mathrm{sec}$, ISO 200, exposure 2). Our detection algorithm for determining the position of the origin line and solvent front line is derived from edge detection algorithms [40] with an assumption that these lines are parallel to the top and bottom edges of the TLC plate. First, we blur the TLC image by filtering the TLC image by a 2D Gaussian filter with sigma of four. This step smooths the image and removes high frequency noise. However, the lines that we need to detect belong to the high frequency part of the image as well. Therefore, we face a tradeoff between blurring and localizing the edges. The tradeoff is determined by the width (sigma) of the Gaussian filter. Sigma of four works well for our dataset in terms of blurring the image without losing the localization of the edges. Also, when applying a 2D Gaussian filter, it is customary to choose a filter size of six sigma with an odd width and height for symmetry with the central peak. With sigma of four, our filter size is 25 by 25 . After smoothing with the Gaussian, we filter the resulting image with the filter $\left[\begin{array}{lll}1 & -2 & 1\end{array}\right]^{\mathrm{T}}$. This step serves the purpose of taking the second derivative of the image in the vertical direction. Since we assume that lines are parallel to the top and bottom edges of the TLC plate, we sum the result of 
the previous step in the horizontal direction in order to make the final localization step easier. In order to localize lines in the last step, we search for the positions of the two strongest local peaks. A local peak is defined as a data sample larger than its two neighbors. The peak near the top of the image corresponds to the solvent front, and the peak near the bottom of the image corresponds to the origin. The front line and origin line detection algorithm is summarized as Algorithm 1 (see the supplementary information for details) and an instance of executing the algorithm is illustrated in Figure 5S(a-d). After locating the origin line and the front line, we crop them from the original image and use the cropped original for the spot detection step.

Spots are comprised of the lowest intensity pixels in a TLC image. Therefore, we choose to employ global thresholding [40], which means using a threshold to convert an image into a binary image. Pixels with values at or above the threshold are assigned a value of 1 , and pixels with values below the threshold are assigned a value of 0 . Global thresholding is simple, fast, and requires minimal computational resources. However, variations in the UV illumination intensity across the plate cause problems with direct application of global thresholding. We correct for this effect by multiplying the image by the inverse of a blank plate image. Figure 5S(e) illustrates the result of this normalization step. Note that some normalized pixels have intensity values greater than unity, which occurs for two reasons. First, limitations of the phone camera such as dynamic range, lack of auto exposure locking, and lack of white balance locking can result in images of a blank plate and a developed plate starting on different $Y$-channel intensity bases. Second, a blank plate may not be an identical replica of the TLC assay plate; thus the blank plate may have slightly different fluorescence intensity. After blank plate correction, we segment out spots by setting a threshold that separates out the darkest $4 \%$ pixels. This threshold was chosen experimentally using several plates in our dataset, then shown to be effective for the remaining plates in the dataset, which results in good visual results for spot segmentation. We further clean up noise in the binary image using a morphological image processing technique. Figure $5 \mathrm{~S}(\mathrm{f})$ illustrates the final result of segmentation.

At this stage, we have a binarized image with spots separated from the background. Spots are then located using a connected component algorithm [40] with eight connectivity, which means that any two pixels are considered connected whenever they are adjacent in horizontal, vertical, or diagonal directions. We then extract data from the resulting components (each is a spot). In order to find the center of a spot, we draw a bounding box around the spot and sum the region in both the vertical and horizontal directions. The center coordinates are located by finding the minima of fitted quadratic curves to those 1D summed signals. We could use the center of the box as the center of the spot, but spots generally appear as ellipses, so fitting gives more accurate estimates of the center. From the center position of each spot, we can derive the spot position value for each spot, representing the distance between the center of the spot and the origin line divided by the distance between solvent front and the origin line. The entire algorithm is summarized as Algorithm 2 (see the supplementary information for details).

The amount of UV light absorbed depends on the amount of API present on the TLC plate, which in turn depends on the concentration of the sample aliquot. Lower concentrations absorb less UV light and produce lighter spots, while higher concentrations absorb more light and produce darker spots. To quantify relative concentration, we integrate the absorption values of the 400 lowest pixel intensities inside the spot. The number 400 was chosen empirically based on our test TLC plates. The absorption value is measured as 1-pixel intensity, where pixel intensity ranges from 0 to 1 . Since the TLC image that we obtain from the camera is in RGB color space, we developed a technique to make use of all channel information. Mathematically, for each pixel of the plate image, the normalized result is a dot product between a three-element vector $\left(A_{1}, A_{2}, A_{3}\right)$ and red, green, blue pixel intensity of the TLC plate subject to the constraint that: 


$$
A_{1} R_{b g}+A_{2} G_{b g}+A_{3} B_{b g}=1
$$

where $R_{b g}, G_{b g}, B_{b g}$ are red, green, blue intensity values of the blank plate. Please refer to Algorithm 3 of the supplementary information that summarizes the spot differentiation process as well as optimal values of $A_{1}, A_{2}$, and $A_{3}$.

\section{Results and discussion}

The smartphone-based TLC analyzer was used to measure two primary characteristics, spot intensity and retention factor $R_{f}$, of a target sample with different concentrations (100\%, 95\%, and $75 \%$ ). Three different drugs (amodiaquine, nevirapine, and paracetamol) were tested to evaluate the system via direct comparison with the analysis provided by the commercial TLC reader.

The analysis results from a plate developed with paracetamol using the smartphone TLC analyzer are shown in Figure 6S. The final processed results are displayed to users by the external algorithm and by the internal algorithm. Drug quality can be evaluated using spot intensity, while drug identity is verified by retention factor. By comparing the spot intensity resulting from the unknown sample to the calibration samples (100\%: standard quality, $75 \%$ : minimum acceptable quality), a decision on whether the unknown sample is acceptable can be rendered. When 95\%-drug is tested among the references, the spot intensity is positioned between those of the reference spots. Whether an unknown sample contains the desired API can be determined by comparing the $R_{f}$ of the unknown with the $R_{f}$ of the calibration samples. For qualitative analysis, there is strong evidence that the drugs in the test and standard solution are identical if the principal spots of both samples show the same $R_{f}$ values. The measurement conducted on the smartphone is guided by the software application's text instructions through the whole process while the analysis implemented on the computer is processed using Matlab software.

Three replicates of a plate loaded with paracetamol were prepared to evaluate the reproducibility of the plate preparation process in which there may be inherent errors resulting from the manual placement of spots (Table 3S). The principal spot intensity and travel distance can vary from one plate to the other due to experimental variants like sample spotting, solvent mixing, and plate development. The coefficient of variation $(C V)$ is defined as $C V=\sigma / \mu$, where the $\sigma$ represents the standard deviation, and $\mu$ is the mean. Both laboratory TLC densitometer and external algorithm yield the same $C V$ for three independent measurements of the $R_{f}$. We obtained $C V$ values of $3 \%, 1.2 \%$, and $1.2 \%$ for $100 \%, 95 \%$, and $75 \%$ drug concentrations, respectively. The $C V$ for the spot intensity measured by the external algorithm for the same spots was $4.9 \%$, $3.0 \%$, and $4.9 \%$ while the $C V$ values were $1 \%, 0.2 \%$, and $1.8 \%$ for the same spots measured with the densitometer. These results are typical for the reproducibility of TLC measurements of plates prepared by a well-trained operator. Generally, we found that the $\mathrm{CV}$ s obtained with the smartphone-based measurement agree well with those obtained with the laboratory TLC densitometer.

Next, we demonstrated the repeatability of our measurements by removing the paracetamol-loaded TLC plate from the cradle and then inserting it again. Figure 3 shows 10 overlaid results from these measurements using both the external and internal algorithms. The external algorithm shows that, when the spot intensity of the $100 \%$ calibration drug is normalized to 1 , a mean spot intensity of $95 \%$ drug is measured to be 0.832 (standard deviation $(\sigma)=0.013$ ) and that of $75 \%$ drug is 0.579 $(\sigma=0.026)$, while the internal algorithm shows $0.939(\sigma=0.001)$ and $0.921(\sigma=0.003)$, respectively. Even though the internal algorithm provides narrow dynamic range for the spot intensity in comparison with the external algorithm, the 
standard deviation is small enough for the internal algorithm to discriminate $5 \%$ difference of the drug quality. In every measurement, the $R_{f}$ values measured by the external algorithm were consistently found to be 0.40 . The $R_{f}$ values processed by the internal algorithms varied from 0.4 to $0.41\left(\sigma_{95 \%}=0, \sigma_{75 \%}=0.001\right)$. The UV lamp output is not robustly stable and the plate is not placed at the exact same position every time, resulting in a small difference in spot intensity between ten independent measurements due to the fact that spot intensity is measured based on pixel intensity analysis. However, the illumination and location do not affect the determined retention factor, and the spot intensity is still proportional to the drug quality. Figure 4 compares the paracetamol spot characteristics from a single TLC plate measured by both the smartphone TLC analyzer and the densitometer. Both algorithms show similar trends of spot intensity reduction as the drug concentration decreases, while the $R_{f}$ values are almost the same. The error bars represent three standard deviations ( $\left.3 \sigma\right)$.

Three drugs were tested and compared using the densitometer and the smartphone TLC analyzer (Table 1). Spot intensities and travel distances measured with the smartphone TLC analyzer were averaged over 10 independent measurements. An analysis of one TLC plate takes 14s for the external algorithm running on a $3.4 \mathrm{GHz}$ Intel i7-2600 processor and 30s for the internal algorithm running on a $1 \mathrm{GHz}$ Cortex-A9 processor, resulting in a one-hour drug test including sample preparation. As shown, both approaches used with the smartphone TLC analyzer provide results similar to those from the commercial TLC reader. Although the non-uniform illumination from the UV lamp is intrinsic to the instrument, the effects of inhomogeneous light distribution are negligible by adopting the normalization processing with a blank TLC plate, as shown in $4 \mathrm{~S}(\mathrm{~d})$ and $5 \mathrm{~S}(\mathrm{e})$. This approach utilizes the entire plate areas, and any $R_{f}$ values between $0 \sim 1$ can be measured regardless of the uneven excitation over the plate. The quality of diluted drugs was distinguishable from that of the standard drug by comparing the normalized spot intensities. In particular, lower drug concentrations produce lower spot intensity values, and the lowest drug concentration provides the smallest spot intensity value. The smartphone TLC analyzer can discern 5\% differences in concentration, as can the densitometer. This is sufficiently high resolution because there is $25 \%$ margin for acceptable drug quality. When falsified pharmaceutical product contains more than $100 \%$ of the API concentration, excess of the API would be reported because the spot intensity would be higher than that of the calibration sample $(100 \%)$. The $R_{f}$ values measured by the external algorithm were the same as those measured by the densitometer, while the internal algorithm gave slightly higher, but consistent $R_{f}$ values to identify the drugs. Each drug shows its own specific $R_{f}$ value. These characteristic $R_{f}$ values can be pre-stored to identify an unknown sample without the use of calibration samples.

\section{Conclusion}

In this work, we have reported the first demonstration of quantitative analysis of pharmaceutical compounds using a mobile phone TLC analyzer. By integrating a UV lamp and the TLC plate in a 3D-printed plastic cradle that can be easily interfaced with a mobile phone, the system serves as a portable scanner for performing TLC plate analysis for identification of falsified and substandard pharmaceutical products. This cost-effective, compact, and portable TLC plate scanning platform integrated with a mobile phone permits direct visualization of principal spots on the plate prepared with fluorescent compound and provides spot intensity and retention factor $R_{f}$ of each spot to facilitate verifying identity and determining an API's quality. We have demonstrated operation of the system by the analysis of three different drugs (nevirapine, amodiaquine, and paracetamol) diluted to $100 \%, 95 \%$ and $75 \%$ of their full concentration. The same approach is applicable to any UV-absorbing API. Two robust image processing frameworks that can be used with an external computer or via an application that runs on an inexpensive smartphone's internal processor were developed to provide a range of options considering the practical tradeoffs in wireless network connectivity, image processing speed, and power consumption. Both 
algorithms were built to overcome the SNR challenges inherent in automated interpretation of TLC spot images, and allow quantitative measurements of principal spots imaged on the smartphone TLC analyzer, achieving discernment of 5\% drug concentration differences, representing the same capability as a laboratory-based desktop TLC densitometer. Although not all drug compounds are UV-active, this mobile phone-based TLC plate scanning platform provides a unique solution for analyzing the contents of UV-absorbing pharmaceutical substances and can be utilized for verifying the authenticity of suspected APIs using a distinct capability that can be widely used in resource-limited settings or remote areas that lack access to a densitometer instrument.

\section{Acknowledgements}

This work was supported by the National Science Foundation (NSF) under Award No. CBET 1264377 and by the Management Sciences for Health (MSH) with funding through the NSF Center for Innovative Instrumentation Technology (CiiT) I/UCRC (IIP 1067943).

\section{Appendix A. Supplementary information}

Illustration of TLC plate development process; mechanical dimensions of 3D-printed cradle; cross section of smartphone TLC analyzer; flow chart of automated chemical constituent quantification (external and internal algorithms); TLC plate image analysis; summary of sample stock and working solution concentrations; mobile phase composition ratio; analysis and comparison of TLC analyzers using paracetamol loaded on three separate plates; chemicals and reagents; processing algorithms of internal algorithm.

\section{References}

[1] V.V. Tolstikov, O. Fiehn, Analysis of Highly Polar Compounds of Plant Origin: Combination of Hydrophilic Interaction Chromatography and Electrospray Ion Trap Mass Spectrometry, Anal. Biochem. 301 (2002) 298-307.

[2] P.J. Taylor, High-performance liquid chromatography-mass spectrometry in the clinical laboratory, Ther. Drug Monit. 27 (2005) 689-693.

[3] A. Stensballe, S. Andersen, O.N. Jensen, Characterization of phosphoproteins from electrophoretic gels by nanoscale Fe(III) affinity chromatography with off-line mass spectrometry analysis, Proteomics 1 (2001) 207-222.

[4] J. Yao, Y.Q. Shi, Z.R. Li, S.H. Jin, Development of a RP-HPLC method for screening potentially counterfeit anti-diabetic drugs, J. Chromatogr. B Analyt. Technol. Biomed. Life Sci. 853 (2007) 254-259.

[5] J. Sherma, Analysis of counterfeit drugs by thin layer chromatography, Acta Chromatogr. 19 (2007) 5-20.

[6] P.Y. Sacre, E. Deconinck, P. Chiap, J. Crommen, F. Mansion, E. Rozet, P. Courselle, J.O. De Beer, Development and validation of a ultra-high-performance liquid chromatography-UV method for the detection and quantification of erectile dysfunction drugs and some of their analogues found in counterfeit medicines, J. Chromatogr. A 1218 (2011) 6439-6447. 
[7] International Medical Products Anti-Counterfeiting Taskforce $\quad$ Web http://www.who.int/impact/FinalBrochureWHA2008a.pdf?ua=1 (accessed Dec 9, 2015).

[8] T.K. Mackey, B.A. Liang, Improving global health governance to combat counterfeit medicines: a proposal for a UNODC-WHO-Interpol trilateral mechanism, BMC Med. 11 (2013) 233.

[9] Africa Renewal Web site. http://www.un.org/africarenewal/magazine/may-2013/counterfeit-drugs-raiseafrica\%E2\%80\%99s-temperature (accessed Dec 9, 2015).

[10] P.E. Wall, Thin-layer chromatography: a modern practical approach, Royal Society of Chemistry, Cambridge, 2005, pp. 6-55.

[11] B. Spangenberg, C.F. Poole, C. Weins, Quantitative thin-layer chromatography - a practical survey, Springer, Heidelberg, 2011, pp. 13-50.

[12] A.N. Zaid, R.J. Al-Ramahi, A. Abu Ghoush, A. Qaddumi, Y. Abu Zaaror, Weight and content uniformity of lorazepam half-tablets: A study of correlation of a low drug content product, Saudi Pharm. J. 21 (2013) 71-75.

[13] D.A. van Riet-Nales, M.E. Doeve, A.E. Nicia, S. Teerenstra, K. Notenboom, Y.A. Hekster, B.J.F. van den Bemt, The accuracy, precision and sustainability of different techniques for tablet subdivision: Breaking by hand and the use of tablet splitters or a kitchen knife, Int. J. Pharmaceut. 466 (2014) 44-51.

[14] I. Elliott, M. Mayxay, S. Yeuichaixong, S.J. Lee, P.N. Newton, The practice and clinical implications of tablet splitting in international health, Trop. Med. Int. Health 19 (2014) 754-760.

[15] J. Zhang, Z. Zhou, J. Yang, W. Zhang, Y. Bai, H. Liu, Thin layer chromatography/plasma assisted multiwavelength laser desorption ionization mass spectrometry for facile separation and selective identification of low molecular weight compounds, Anal. Chem. 84 (2012) 1496-1503.

[16] B. Fuchs, R. Suss, K. Teuber, M. Eibisch, J. Schiller, Lipid analysis by thin-layer chromatography: a review of the current state, J. Chromatogr. A 1218 (2011) 2754-2774.

[17] S. Buchinger, D. Spira, K. Broder, M. Schlusener, T. Ternes, G. Reifferscheid, Direct coupling of thin-layer chromatography with a bioassay for the detection of estrogenic compounds: applications for effect-directed analysis, Anal. Chem. 85 (2013) 7248-7256.

[18] Chemotron Inc. Web site. http://www.chemotron.hu/chemotron_en.htm (accessed Dec 9, 2015).

[19] Chemeurope Web site. http://www.chemeurope.com (accessed Dec 9, 2015).

[20] CAMAG Web site. http://www.camag.com (accessed Dec 9, 2015).

[21] eMarketer Web site. http://www.emarketer.com/Article/2-Billion-Consumers-Worldwide-Smartphones-by2016/1011694 (accessed Dec 9, 2015).

[22] Gartner Web site. http://www.gartner.com/newsroom/id/2996817 (accessed Dec 12, 2015).

[23] S.Q. Wang, X.H. Zhao, I. Khimji, R. Akbas, W.L. Qiu, D. Edwards, D.W. Cramer, B. Ye, U. Demirci, Integration of cell phone imaging with microchip ELISA to detect ovarian cancer HE4 biomarker in urine at the point-of-care, Lab Chip 11 (2011) 3411-3418.

[24] C.M. McGeough, S. O'Driscoll, Camera Phone-Based Quantitative Analysis of C-Reactive Protein ELISA, IEEE T. Biomed. Circ. S. 7 (2013) 655-659.

[25] D. Gallegos, K.D. Long, H.J. Yu, P.P. Clark, Y.X. Lin, S. George, P. Nath, B.T. Cunningham, Label-free biodetection using a smartphone, Lab Chip 13 (2013) 2124-2132. 
[26] H.Y. Zhu, S. Mavandadi, A.F. Coskun, O. Yaglidere, A. Ozcan, Optofluidic Fluorescent Imaging Cytometry on a Cell Phone, Anal. Chem. 83 (2011) 6641-6647.

[27] H.J. Yu, Y.F. Tan, B.T. Cunningham, Smartphone Fluorescence Spectroscopy, Anal. Chem. 86 (2014) $8805-8813$.

[28] E. Petryayeva, W.R. Algar, Single-step bioassays in serum and whole blood with a smartphone, quantum dots and paper-in-PDMS chips, Analyst 140 (2015) 4037-4045.

[29] C.G. Scully, J. Lee, J. Meyer, A.M. Gorbach, D. Granquist-Fraser, Y. Mendelson, K.H. Chon, Physiological Parameter Monitoring from Optical Recordings With a Mobile Phone, IEEE Trans. Biomed. Eng. 59 (2012) 303-306.

[30] S. Dutta, D. Sarma, P. Nath, Ground and river water quality monitoring using a smartphone-based pH sensor, Aip Adv. 5 (2015) 057151 .

[31] W. Zhang, A. Jones, M. Doherty, Does paracetamol (acetaminophen) reduce the pain of osteoarthritis?: a meta-analysis of randomised controlled trials, Ann. Rheum. Dis. 63 (2004) 901-907.

[32] A. Violari, J.C. Lindsey, M.D. Hughes, H.A. Mujuru, L. Barlow-Mosha, P. Kamthunzi, B.H. Chi, M.F. Cotton, H. Moultrie, S. Khadse, W. Schimana, R. Bobat, L. Purdue, S.H. Eshleman, E.J. Abrams, L. Millar, E. Petzold, L.M. Mofenson, P. Jean-Philippe, P. Palumbo, Nevirapine versus Ritonavir-Boosted Lopinavir for HIV-Infected Children, New Engl. J. Med. 366 (2012) 2380-2389.

[33] S.B. Sirima, A. Gansane, Artesunate-amodiaquine for the treatment of uncomplicated malaria, Expert Opin. Inv. Drug. 16 (2007) 1079-1085.

World

Health

Organization

Web

site.

http://www.who.int/selection_medicines/committees/expert/20/EML_2015_FINAL_amended_JUN2015.pdf?ua=1 (accessed Dec 12, 2015).

[35] World Health Organization Web site. http://apps.who.int/phint/en/p/docf (accessed Dec 12, 2015).

[36] GPHF-Minilab Web site. http://www.gphf.org/en/minilab (accessed Dec 12, 2015).

[37] X. Wu, YIQ vector quantization in a new color palette architecture, IEEE Trans. Image Process. 5 (1996) $321-329$.

[38] M. Bigas, E. Cabruja, J. Forest, J. Salvi, Review of CMOS image sensors, Microelectron. J. 37 (2006) $433-451$.

[39] P. Zhou, K.E. Goodson, Subpixel displacement and deformation gradient measurement using digital image/speckle correlation (DISC), Opt. Eng. 40 (2001) 1613-1620.

[40] R.C. Gonzalez, R.E. Woods, Digital image processing, third ed., Prentice Hall, New Jersey, 2008, pp. 657-761. 
Figure 1: Illustration of the smartphone TLC analyzer and photographs of the system. $(a, b)$ Illustration of the cradle that holds a smartphone, a UV lamp, and a TLC plate. (c) Illustration of the cover provides a dark environment and that mechanically supports the lamp. (d) Photo of the assembled smartphone TLC analyzer. (e) Photo of the TLC plate analysis (image capturing/processing). (f) Captured full image of the TLC plate.

Figure 2. TLC plate images after development. (a) Visible light. (b) With UV illumination. (c) Distribution of fluorescence intensity.

Figure 3. Results from 10 independent measurements of a paracetamol TLC plate taken by removing and re-inserting the plate into the cradle, demonstrating excellent measurement reproducibility. Normalized spot intensities and travel distances $\left(R_{f}\right)$ measured by the external algorithm (a, b) and by the internal algorithm (c, d).

Figure 4. Normalized spot intensity/travel distance $\left(R_{f}\right)$ measurements and comparison with measurements of the same plate taken with a densitometer for a TLC plate prepared with paracetamol spots. 
Figure 1

(a)

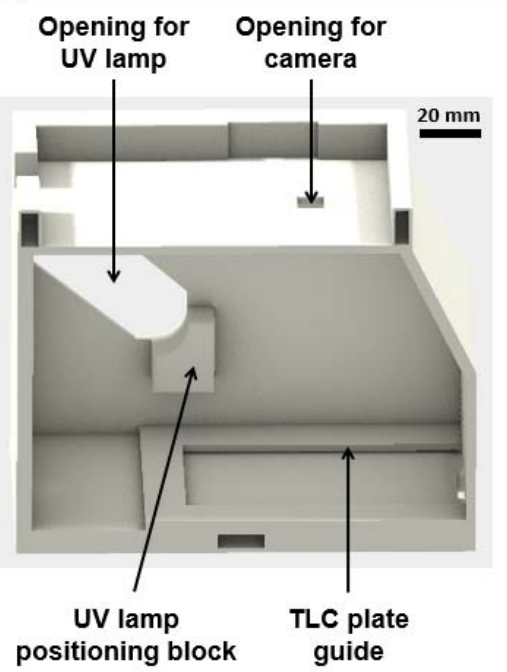

(b)

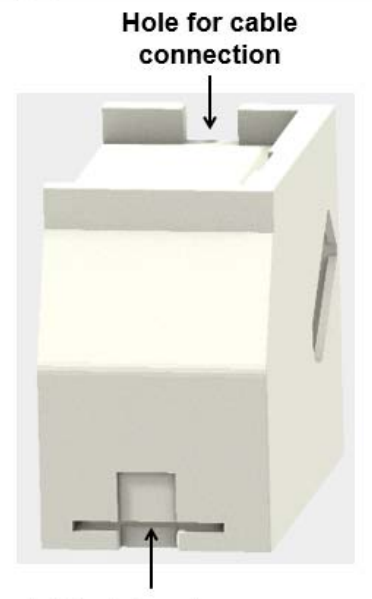

TLC plate entrance (c)

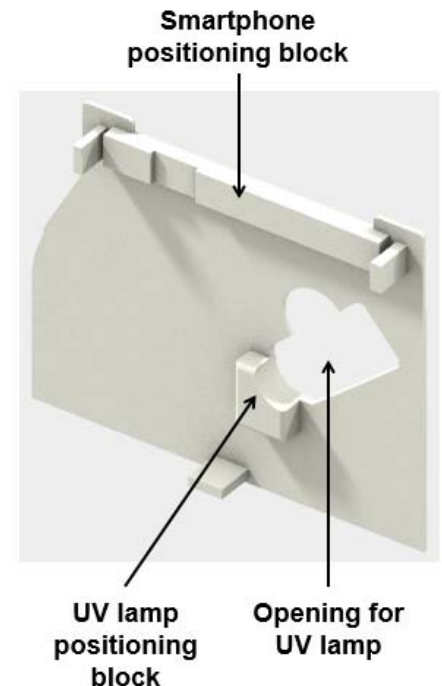

(d)

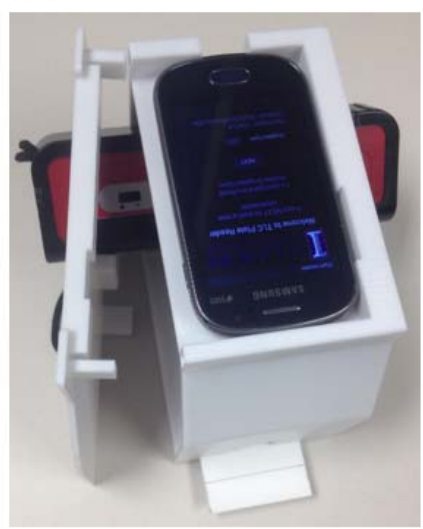

(e)

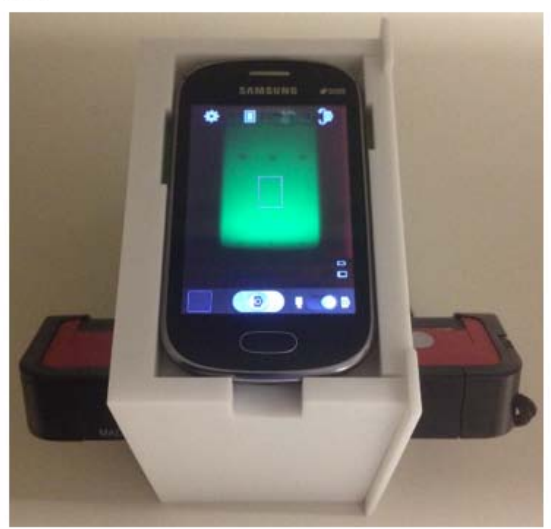

(f)

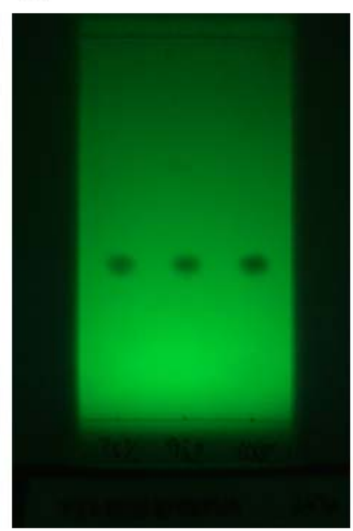


Figure 2

(a)

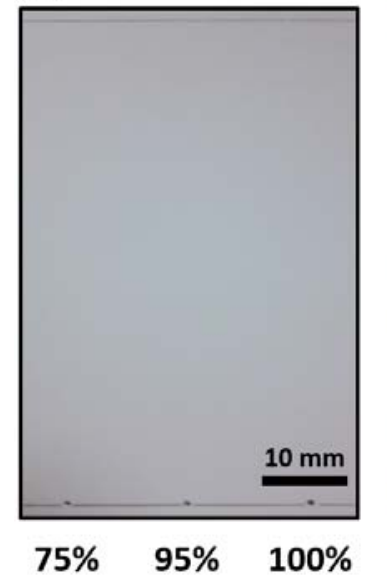

Drug concentrations (b)

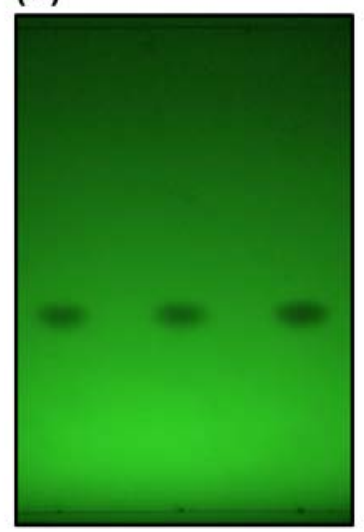

(c)

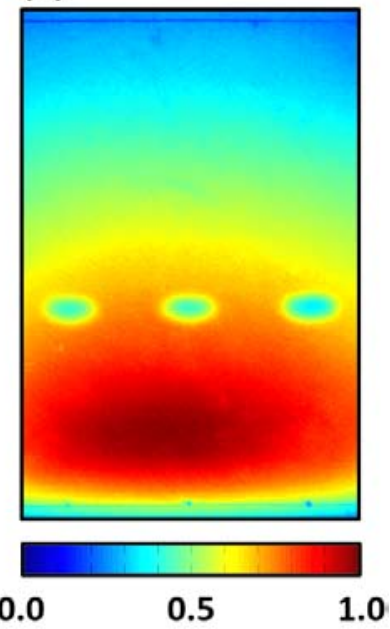


Figure 3

(a)

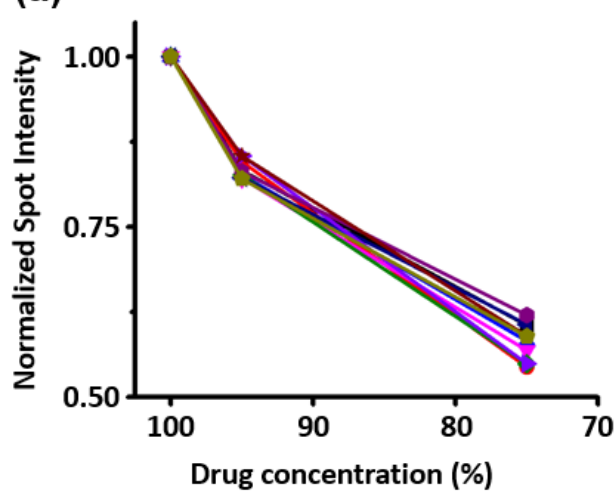

(c)

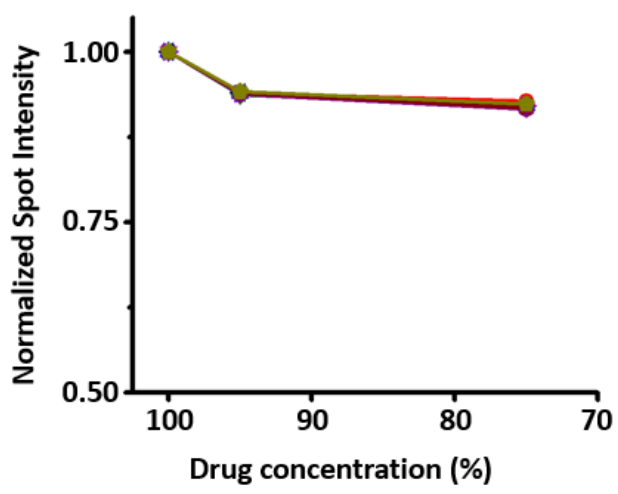

(b)

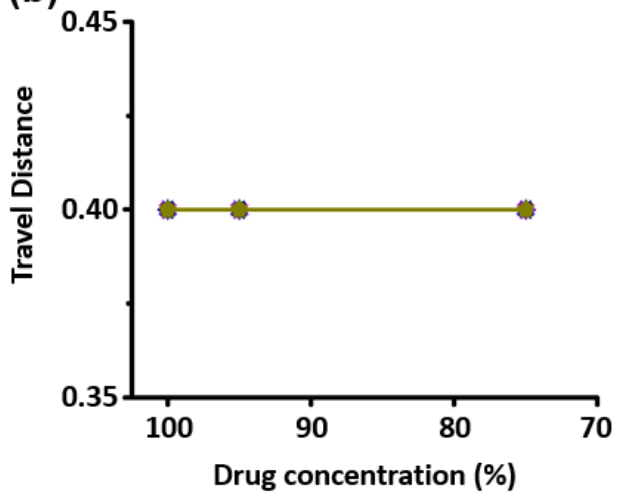

(d)

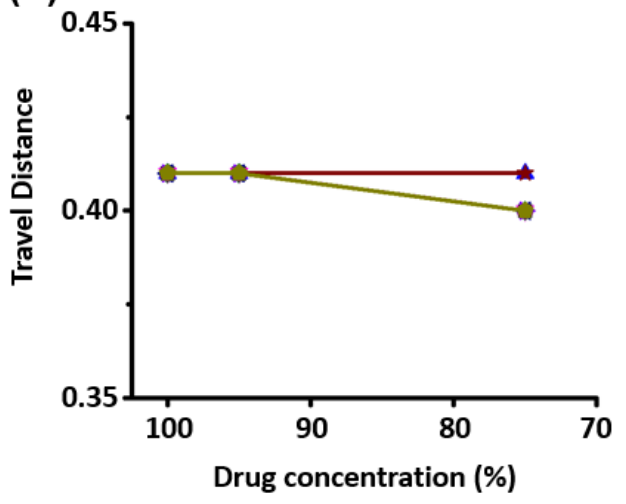

\begin{tabular}{|lll|}
\hline- Measurement \#1 & - Measurement \#5 & - Measurement \#8 \\
- - Measurement \#2 & - Measurement \#6 & - Measurement \#9 \\
- & - Measurement \#3 & - Measurement \#10 \\
$-\checkmark$ - Measurement \#4 & & \\
\hline
\end{tabular}


Figure 4
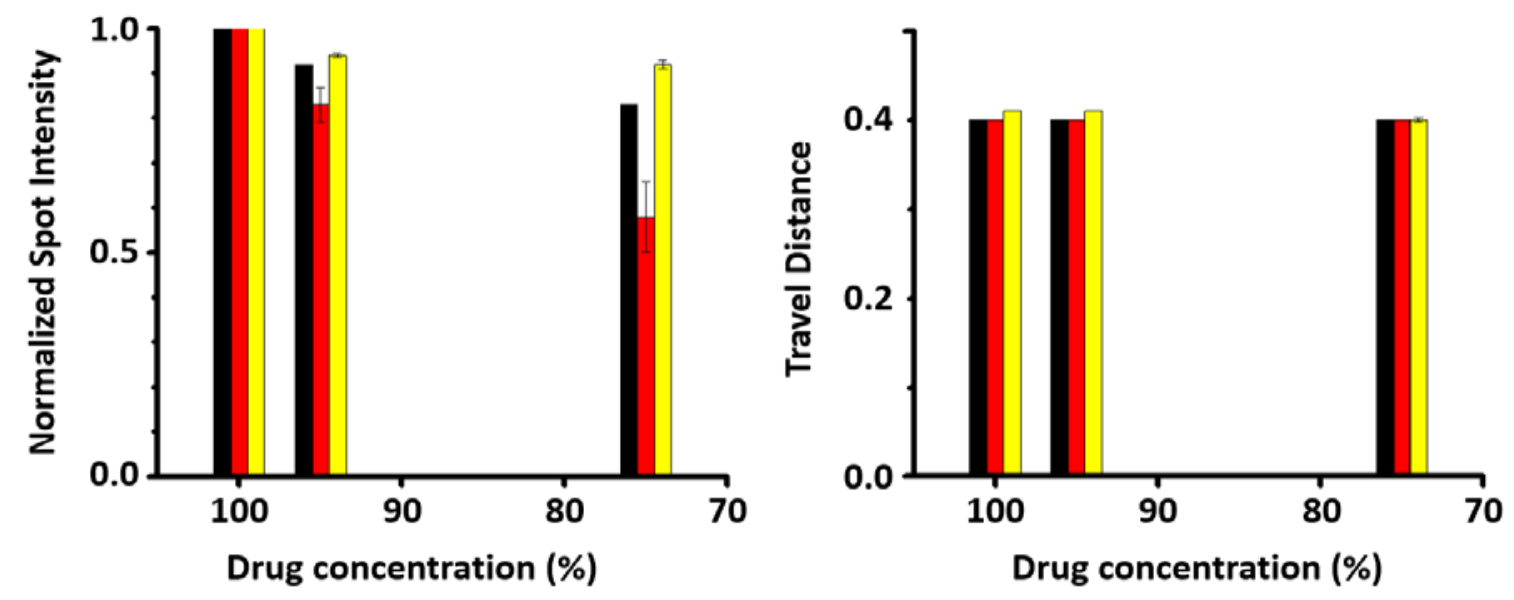

Densitometer External algorithm Internal algorithm 
Table 1. Analysis and comparison of TLC analyzers using three different drugs

\begin{tabular}{|c|c|c|c|c|c|}
\hline \multirow{2}{*}{ Drugs } & \multirow{2}{*}{ Measurements } & \multirow{2}{*}{ Drug Conc. } & \multirow{2}{*}{ Densitometer } & \multicolumn{2}{|c|}{ Smartphone TLC Analyzer } \\
\hline & & & & External & Internal \\
\hline \multirow{6}{*}{ Amodiaquine } & \multirow{3}{*}{$\begin{array}{l}\text { Normalized } \\
\text { Spot Intensity }\end{array}$} & $100 \%$ & 1 & 1 & 1 \\
\hline & & $95 \%$ & 0.958 & 0.906 & 0.980 \\
\hline & & $75 \%$ & 0.932 & 0.755 & 0.941 \\
\hline & \multirow{3}{*}{$\begin{array}{c}\text { Travel Distance } \\
\qquad\left(R_{f}\right)\end{array}$} & $100 \%$ & 0.73 & 0.73 & 0.74 \\
\hline & & $95 \%$ & 0.73 & 0.73 & 0.74 \\
\hline & & $75 \%$ & 0.73 & 0.73 & 0.73 \\
\hline \multirow{6}{*}{ Nevirapine } & \multirow{3}{*}{$\begin{array}{l}\text { Normalized } \\
\text { Spot Intensity }\end{array}$} & $100 \%$ & 1 & 1 & 1 \\
\hline & & $95 \%$ & 0.938 & 0.817 & 0.974 \\
\hline & & $75 \%$ & 0.806 & 0.771 & 0.947 \\
\hline & \multirow{3}{*}{$\begin{array}{c}\text { Travel Distance } \\
\qquad\left(R_{f}\right)\end{array}$} & $100 \%$ & 0.61 & 0.61 & 0.62 \\
\hline & & $95 \%$ & 0.61 & 0.61 & 0.62 \\
\hline & & $75 \%$ & 0.61 & 0.61 & 0.62 \\
\hline \multirow{6}{*}{ Paracetamol } & \multirow{3}{*}{$\begin{array}{c}\text { Normalized } \\
\text { Spot Intensity }\end{array}$} & $100 \%$ & 1 & 1 & 1 \\
\hline & & $95 \%$ & 0.919 & 0.832 & 0.939 \\
\hline & & $75 \%$ & 0.831 & 0.579 & 0.921 \\
\hline & \multirow{3}{*}{$\begin{array}{c}\text { Travel Distance } \\
\qquad\left(R_{f}\right)\end{array}$} & $100 \%$ & 0.40 & 0.40 & 0.41 \\
\hline & & $95 \%$ & 0.40 & 0.40 & 0.41 \\
\hline & & $75 \%$ & 0.40 & 0.40 & 0.40 \\
\hline
\end{tabular}

